\title{
Microbiologically proven bacterial infections in AIDS
}

\author{
BL Kirkpatrick, SC Glover, DS Reeves, AP MacGowan
}

\begin{abstract}
Summary
We have reviewed the incidence, type and site of microbiologically proven bacterial infection occurring in 52 patients with the acquired immunodeficiency syndrome (AIDS) who presented to Southmead Hospital, Bristol between 1990 and 1994. A total of 30 (58\%) patients had significant bacterial isolates. The majority of infections were community acquired. Overall, more infections were caused by Gramnegative organisms but Gram-positive organisms predominated in bacteraemia. Mycobacterium avium intracellulare (MAI) caused infection in the largest number of patients, followed by Staphylococcus aureus, Streptococcus pneumoniae, Pseudomonas sp, and Campylobacter sp. When individual episodes of infection were considered, after MAI, Haemophilus infuenzae, Streptococcus pneumoniae and Pseudomonas sp were the organisms most frequently isolated; often these same organisms caused recurrent chest infection. Bacterial infections in AIDS patients are common and although they generally respond well to antimicrobial chemotherapy there is a high recurrence rate, particularly in the respiratory tract, which is the commonest site of infection.
\end{abstract}

Keywords: acquired immunodeficiency syndrome, bacterial infection

The acquired immune deficiency syndrome (AIDS) is caused by human immunodeficiency virus (HIV) infection, the main abnormality of which is immunosuppression. Although $T$ lymphocyte dysfunction gives rise to infection with opportunist organisms, B lymphocyte dysfunction and neutrophil abnormalities (both of number and function) lead to infection with conventional bacterial pathogens. In children, bacterial infections are the commonest manifestation of immunodeficiency due to HIV. ${ }^{1}$ Adults with HIV disease have a higher frequency of bacterial infection than the general population ${ }^{1}$ and serious nonopportunist bacterial infections are recognised more frequently than in the past. ${ }^{2}$

It is important to be aware of the pattern and incidence of infections associated with AIDS and so we have reviewed the bacterial infections which have occurred in 52 AIDS patients admitted to a district general hospital in order to identify the rate, type and site of infection occurring in these patients.

\section{Methods}

Patients who presented to the Infectious Diseases Service at Southmead Hospital, Bristol and were notifed as having AIDS during the period 1990-94 were included in the review. Data were collected from Communicable Disease Surveillance Centre notifications of AIDS in order to discover patient age, sex, length of AIDS illness and mortality. Information on the source of HIV infection as well as the AIDS-defining diagnosis was also obtained. Data were collected on the patients' bacterial isolates and in those patients with potentially significant bacterial isolates the hospital notes were reviewed to assess the isolate's clinical importance. Only those patients who had clinically significant isolates after the diagnosis of AIDS was made were included in the review. Data were collected on the type of organism and site of infection, the source of infection (whether community acquired or not), therapy and outcome. Peripheral blood white cell counts and CD4 counts for each individual episode of infection were also recorded.

\section{Results}

Thirty patients ( $57.7 \%$ of patients studied) had bacterial isolates which were judged as having clinical significance (table 1). Mycobacterium avium intracellulare (MAI) was the organism which caused infection in the largest number of patients. If the number of episodes of infection caused by each individual organism per 'at risk' patient per year are considered, the highest rate is caused by MAI $(0.16)$, followed by $H$ influenzae (0.14), $S$ pneumoniae (0.13) and Pseudomonas sp (0.1). A large number of episodes of infection caused by $H$ influenzae and $S$ pneumoniae were related to four patients who had recurrent chest infections caused by these organisms.

\section{AGE, SEX, MORTALITY AND PERIPHERAL BLOOD} CD4 COUNT

Most of the 30 patients with significant bacterial isolates reviewed were male (27). The age range was $23-51$ years, with a mean of 36.6 at the time when AIDS was diagnosed. Of the 30 with bacterial infection, three were still alive at the time of the review (and one was 
Table 1 Bacterial isolates from AIDS patients

\begin{tabular}{|c|c|c|c|}
\hline Isolate & $\begin{array}{l}\text { Number of } \\
\text { patients } \\
\text { infected } \\
(n=30)^{*}\end{array}$ & $\begin{array}{l}\text { Number of } \\
\text { episodes of } \\
\text { bacterial } \\
\text { infection }\end{array}$ & $\begin{array}{l}\text { Number of } \\
\text { episodes } \\
\text { per patient } \\
\text { year }\end{array}$ \\
\hline Staphylococcus aureus & 4 & $4^{\star \star}$ & 0.06 \\
\hline Coagulase-negative staphylococci & 3 & 3 & 0.04 \\
\hline Streptococcus pneumoniae & 4 & 9 & 0.13 \\
\hline Enterococcus faecalis & 3 & 6 & 0.09 \\
\hline Enterococcus faecium & 1 & 1 & 0.01 \\
\hline Diphtheroid sp & 1 & 1 & 0.01 \\
\hline Bacillus carotarum & 1 & 1 & 0.01 \\
\hline Pseudomonas sp & 4 & $7 \dagger$ & 0.1 \\
\hline Escherichia coli & 1 & 2 & 0.03 \\
\hline Coliforms & 2 & $3+\dagger$ & 0.04 \\
\hline Salmonella $\mathrm{sp}$ & 2 & 2 & 0.03 \\
\hline Shigella sonnei & 1 & 1 & 0.01 \\
\hline Campylobacter sp & 4 & 4 & 0.06 \\
\hline Haemophilus influenzae & 2 & 10 & 0.14 \\
\hline Moraxella catarrhalis & 3 & 3 & 0.04 \\
\hline Mixed bacteria from urine & 1 & 2 & 0.03 \\
\hline Mixed anaerobes & 1 & 1 & 0.01 \\
\hline Acid-fast bacilli & 1 & 1 & 0.01 \\
\hline Mycobacterium kansasii & 2 & 2 & 0.03 \\
\hline Mycobacterium avium intracellulare & 11 & 11 & 0.16 \\
\hline
\end{tabular}

* Some patients were infected on more than one occasion; ${ }^{\star \star}$ four episodes but five sites culture positive, as blood cultures and MSU positive at the same time; $†$ seven episodes but nine isolates as blood cultures and Hickman line site culture positive at the same time on two occasions; ††three coliform urinary tract infections of which one resulted in $E$ coli bacteraemia
Table 2 AIDS-defining illnesses in those who developed bacterial infection

\begin{tabular}{ll}
\hline AIDS-defining illness & $\begin{array}{l}\text { No of } \\
\text { patients } \\
(n=30)\end{array}$ \\
\hline $\begin{array}{l}\text { Pneumocystis carinii pneumonia (PCP) } \\
\text { PCP and Mycobacterium avium }\end{array}$ & 10 \\
$\quad$ intracellulare (MAI) & 1 \\
PCP and Kaposi's sacrcoma & 1 \\
Kaposi's sarcoma & 3 \\
Kaposi's and chronic cryptosporidia & 1 \\
MAI & 3 \\
MAI and wasting syndrome due to HIV & 1 \\
MAI and cytomegalovirus (CMV) & 1 \\
$\quad$ retinitis & 1 \\
CMV chest infection & 1 \\
Recurrent chest infection & 4 \\
Oesophageal candidiasis & 1 \\
Chronic diarrhoea due to cryptosporidia & 1 \\
HIV encephalopathy & 2
\end{tabular}

lost to follow-up). The time from the diagnosis of AIDS to death ranged from 1-51 months (mean of 18.1 months; data available for 24 patients). Where CD4 counts were documented at the time of each infection, these were $\leqslant 0.1 \times 10^{9} / 1$, in all but one patient.

SOURCE OF HIV INFECTION AND AIDS-DEFINING ILLNESS

Twenty-one patients were homosexual, of whom one was also an intravenous drug user and one also had had sexual intercourse abroad. Four were heterosexual patients who had had sexual intercourse abroad, all in Africa. A further two heterosexual patients were intravenous drug users, one woman had a partner who abused intravenous drugs while another woman had a bisexual partner. In one patient the risk factor for contracting HIV was not known. The AIDS-defining illnesses are given in table 2 .

\section{SITE OF INFECTION}

If mycobacterial infections are excluded, most patients had respiratory tract infection (11) followed by gastrointestinal tract infection (7), urinary tract infection (5) and bacteraemia (5) (table 3 ). Where actual episodes of infection are considered and the rate of infectious episode per 'at risk' patient year calculated, the highest rates were still recorded for respiratory tract infections $(0.37)$, followed by urinary tract infection $(0.17)$, bacteraemia (0.13) and gastrointestinal tract infection (0.1) (table 3). Patients with recurrent infection account for this alteration in order when episodes per patient year are compared with individual patient rates.

\section{BACTERAEMIA}

Five patients had positive blood cultures on nine separate occasions, and on eight occasions patients were actively treated with appropriate antimicrobial therapy (table 4). One patient who was terminally ill had treatment withheld. Two patients had multiple episodes of bacteraemia, the first due to $E$ coli and then $S$ aureus, and the second patient due to $S$ pneumoniae, $S$ aureus and then two episodes of Ps aeruginosa bacteraemia. The source of infection for these bacteraemias was presumed to be lung in one patient ( $S$ pneumoniae), urine in one patient $(E$ coli), Hickman line in five (Ps aeruginosa-two episodes in one patient, Diphtheroid sp, coagulase-negative staphylococci and $E$ faecium). 23 months after the patients' diagnosis of AIDS was made (range 9-29 months, seven observations). Eight episodes of bacteraemia were acquired in the community and only one ( $E$ faecalis) appeared to be hospital acquired.

\section{BACTERIAL GASTROENTERITIS}

Seven patients had enteric pathogens isolated on stool culture, of which six were of definite clinical significance. One patient had a Campylobacter upsaliensis isolated from two specimens and although a history of loose stool was recorded, its clinical significance was difficult to assess. Three other patients had Campylobacter $\mathrm{sp}$ isolated from faeces. One patient also had a co-existent infection with Cryptosporidia. Another patient with Campylobacter sp improved after five days therapy with ciprofloxacin but a third died one week following isolation of Campylobacter sp, despite appropriate antimicrobial therapy. However, it is likely that this patient had other ongoing infection which contributed to his death (see below). The time taken from the AIDS diagnosis to Campylobacter infection ranged between two and 16 months (mean eight months). Two patients developed salmonella infections ( $S$ chester and $S$ enteriditis) and both responded to therapy with ciprofloxacin. These patients both developed these infections at the
Positive blood cultures occurred on average 
same time as their diagnosis of AIDS was made. The last patient with gastrointestinal infection was a woman who developed Shigella sonneii infection which again responded to ciprofloxacin. Shigella infection developed five months after her diagnosis of AIDS was made.

Table 3 Sites of bacterial infections and incidence of infection

\begin{tabular}{lll}
\hline & $\begin{array}{l}\text { Number of patients } \\
\text { notified as having } \\
\text { AIDS who had } \\
\text { bacterial infection } \\
(n=52)(\%)\end{array}$ & $\begin{array}{l}\text { Episodes per } \\
\text { patient year }\end{array}$ \\
& $30(57.7 \%)$ & 1.06 \\
All bacterial infections & $5(9.6 \%)$ & 0.13 \\
Bacteraemia & $2(3.8 \%)$ & \\
$\quad$ multiple episodes of bacteraemia & $7(13.5 \%)$ & 0.1 \\
Bacterial gastroenteritis & $2(3.8 \%)$ & 0.03 \\
Skin and soft tissue infection & $2(3.8 \%)$ & 0.06 \\
Line-related infection & $1(1.9 \%)$ & 0.37 \\
$\quad$ recurrent line-related infection & $11(21.1 \%)$ & 0.17 \\
Respiratory tract infection & $5(9.6 \%)$ & \\
$\quad$ recurrent respiratory tract infection & $5(9.6 \%)$ & 0.19 \\
Urinary tract infection & $3(5.8 \%)$ & 0.01 \\
$\quad$ recurrent urinary tract infection & $13(25 \%)$ & \\
Mycobacterial infection (all sites) & $1(1.9 \%)$ & \\
Other infection & &
\end{tabular}

*Some patients had more than infection

Table 4 Sites of infection and bacterial isolates

\begin{tabular}{|c|c|c|}
\hline Site of infection & Isolates & $\begin{array}{l}\text { Number of } \\
\text { episodes }\end{array}$ \\
\hline \multirow{7}{*}{ Bacteraemia } & Staphylococcus aureus & 2 \\
\hline & Enterococcus faecalis & 1 \\
\hline & Enterococcus faecium & 1 \\
\hline & Diphtheroid sp & 1 \\
\hline & $\begin{array}{l}\text { Streptococcus pneumoniae }+ \\
\quad \text { coagulase-negative staphylococci }\end{array}$ & 1 \\
\hline & Escherichia coli & 1 \\
\hline & Ps aeruginosa ${ }^{\star}$ & 2 \\
\hline \multirow[t]{3}{*}{ Gastrointestinal tract infection } & Campylobacter sp & 4 \\
\hline & Salmonella $\mathrm{sp}$ & 2 \\
\hline & Shigella sonnei & 1 \\
\hline \multirow[t]{2}{*}{ Skin and soft tissue infection } & $\begin{array}{l}\text { Mixed anaerobes and coagulase- } \\
\text { negative staphylococci }\end{array}$ & 1 \\
\hline & Ps aeruginosa & 1 \\
\hline \multirow[t]{2}{*}{ Line-related infection } & Ps aeruginosa ${ }^{\star}$ & 3 \\
\hline & Coagulase-negative staphylococci & 1 \\
\hline \multirow[t]{5}{*}{ Respiratory tract infection } & $H$ influenzae $e^{\star}$ & 10 \\
\hline & $S$ pneumoniae ${ }^{\star}$ & 8 \\
\hline & Moraxella catarrhalis & 3 \\
\hline & $S$ aureus & 2 \\
\hline & Ps aeruginosa ${ }^{\star}$ & 3 \\
\hline \multirow[t]{5}{*}{ Urinary tract infection } & Enterococcus faecalis * & 5 \\
\hline & coliforms & 3 \\
\hline & $E$ coli & 1 \\
\hline & $S$ aureus & 1 \\
\hline & Mixed growth ${ }^{\star}$ & 2 \\
\hline \multirow[t]{4}{*}{ Mycobacterial infection } & MAI & 10 \\
\hline & MAI and Mycobacterium kansasii & 1 \\
\hline & M kansasii & 1 \\
\hline & Acid-fast bacilli only & 1 \\
\hline Other infection & Bacillus carotarum & 1 \\
\hline
\end{tabular}

${ }^{\star}$ Recurrent infection accounted for some of these episodes
SKIN AND SOFT TISSUE INFECTION

Two patients had significant infection from which a bacterial diagnosis was made. The first developed infected facial sores which were treated empirically with anti-staphylococcal therapy but then these progressed and he became systemically unwell. A diagnosis of ecthyma gangrenosum was made and $P s$ aeruginosa was grown from several sites on face and chest. This patient had other significant infections which included $S$ chester enteritis and $E$ faecium bacteraemia.

The second patient who had anaerobes and coagulase-negative staphylococci isolated from scrotal pus at post mortem, was admitted two months before death when he was found to have a rectovesical fistula, scrotal abscess, cellulitis and chronic sacral osteomyelitis. $\mathrm{He}$ also had two urine cultures with mixed organisms including anaerobic cocci which supported the diagnosis of rectovesical fistula and at the same time had a Campylobacter $\mathrm{sp}$ isolated from a faecal specimen (see above).

\section{HICKMAN LINE INFECTIONS}

One patient had Pseudomonas sp isolated from his Hickman line exit site on two occasions five weeks apart and simultaneously had Pseudomonas $\mathrm{sp}$ isolated from blood cultures at both these times. His line was removed following the second episode. Pseudomonas sp was again isolated from his Hickman line site five months later.

The significance of coagulase-negative staphylococci grown from a Hickman entry site in an intravenous drug abuser with a fever and treated with vancomycin has not been established as this patient also suffered from disseminated MAI infection which was being treated simultaneously.

\section{RESPIRATORY TRACT INFECTIONS}

Eleven patients had significant respiratory tract isolates and of these five had recurrent infections, (two episodes of Pseudomonas sp infection, three episodes of $H$ influenzae and one episode of $S$ pneumoniae, two episodes of $S$ pneumoniae infection, five episodes of $S$ pneumoniae and seven episodes of $H$ influenzae infection, respectively). The two patients with recurrent $H$ influenzae infection both survived all their $H$ influenzae infections. One had three episodes of $H$ influenzae infection which responded firstly to co-amoxiclav; four months later he developed a clinically and bronchoscopically confirmed chest infection with $H$ influenzae and was treated with clarithromycin but he required re-admission 12 days later for a course of intravenous ciprofloxacin as $H$ influenzae was still being isolated. Three weeks later he developed a right basal bronchopneumonia due to $S$ pneumoniae and despite appropriate therapy he died. The second patient had nine isolates of $H$ influenzae from sputum over a period of 16 months, seven of which were thought to have been clinically significant and were actively treated. His therapy included three courses of oral cephalosporins, four courses of parenteral cephalosporins, three courses of ciprofloxacin, two 
courses of erythromycin, clarithromycin, oxytetracycline, and rifampicin as well as longterm cefixime and ceftriaxone.

Three patients had documented $S$ pneumoniae infections, two of whom had recurrent infections. All were male and two were intravenous drug abusers. One patient had five episodes which occurred over a 21-month period and all episodes were appropriately treated. Following this it was planned that he should have long-term therapy rotating coamoxiclav with clarithromycin. A further patient who had two episodes of $S$ pneumoniae infection recovered from the first after a course of co-amoxiclav only to be re-admitted the following month with another episode which eventually responded to a third generation cephalosporin. $M$ kansasii was also found in his sputum and blood at this time and he died shortly afterwards. The third patient was one who had recurrent $H$ influenzae infection and then had an episode of $S$ pneumoniae infection from which he died.

$M$ catarrhalis was isolated from sputum or bronchoalveolar lavages of three patients, all of whom had Kaposi's sarcoma. $S$ aureus was isolated from two patients each in mixed growth with Candida sp. Two patients acquired clinically significant Ps aeruginosa infections, the first of whom had HIV encephalopathy diagnosed three months earlier. The second patient had two episodes of Pseudomonas infection two years apart.

Two out of the total 26 episodes of pulmonary infection were nosocomially acquired (Pseudomonas sp and $M$ catarrhalis).

\section{SIGNIFICANT BACTERIURIA}

Urinary tract infections were difficult to review as it was not always clear from the notes whether the patient was symptomatic, the isolate was interpreted as being clinically significant or whether therapy was prescribed. In all, 12 urine samples (the majority of which were MSU samples) from five patients had signficant bacteriuria. Five isolates were appropriately treated and two patients were on antimicrobial therapy for other infections which would also have been active against the urinary isolates. The 12 isolates included $E$ faecalis (5), coliforms (3), $S$ aureus (1), E coli (1), mixed growth (two samples from a single patient with a rectovesical fistula). Two of these isolates were assumed to be hospital acquired.

\section{MYCOBACTERIAL INFECTIONS}

Thirteen patients had microbiologically documented mycobacterial infections, 10 of which were due to MAI alone, one had both MAI plus $M$ kansasii infection and one $M$ kansasii alone. The thirteenth patient had a liver biopsy in which acid-fast bacilli were seen and although these were not subsequently grown, this was treated as a definite infection.

For the 10 patients who had MAI alone, all isolates were grown from blood samples, five also grew from bone marrow and two from faeces. The patient with $M$ kansasii alone had it in both sputum and blood, and the patient with a mixed infection of $M$ kansasii and MAI had the former in blood, abdominal ulcer and rectal specimens and the latter in sputum. Twelve patients died at a mean time of 8.6 months (range 1-32 months) after the infection was diagnosed and in one patient the diagnosis was only made after death. Nine of these patients had no other bacterial infections. The other four patients' bacterial infections included pneumococcal chest infections (1), $S$ enteriditis from faeces (1), recurrent bacteraemia (1) and $S$ aureus chest infection (1). For six, the mycobacterial infection was the AIDSdefining illness and in seven it occurred from 1 to 51 months (mean 18.6 months) after the diagnosis of AIDS was made.

\section{OTHER INFECTION}

Bacillus carotarum was isolated from liver biopsy in a patient who was diagnosed as having AIDS 23 months earlier. It was regarded as clinically significant and he received appropriate antimicrobial chemotherapy.

\section{NEUTROPENIA}

Only four patients were neutropenic (neutrophils $<1.0 \times 10^{9} \mathrm{~g} / \mathrm{l}$ ) at the time a significant bacterial isolation was made. This may be an underestimate as neutrophil counts were not available for all. The isolates which were documented at the time of the neutropenia included $C$ upsaliensis enteric infection, Pseudomonas sp from blood cultures, Pseudomonas $\mathrm{sp}$ from skin and tissue samples from the patient with ecthyma gangrenosum and an $E$ faecalis urinary tract infection. Only two of the patients were on zidovudine at this time and in addition one had completed chemotherapy and radiotherapy for Kaposi's sarcoma.

\section{Discussion}

By 1994 over 9000 cases of AIDS had been notified in the UK. ${ }^{3}$ We have reviewed microbiologically proven bacterial infections in a group of 52 AIDS patients who presented to a district general hospital. The majority of patients in this review were homosexual males who were at an advanced stage in their disease with CD4 counts less than $0.1 \times 10^{9} / 1$.

In this group of patients the commonest AIDS-defining illness was that of PCP followed by MAI, Kaposi's sarcoma, and oesophageal candidiasis. With severe T-cell defects and dysfunction in patients such as these, infection due to atypical mycobacteria (predominantly MAI) is the commonest type of bacterial infection. Disseminated infection with MAI tends to occur late in the course of HIV infection. ${ }^{4}$ As these patients all had AIDS and were therefore reviewed at a late stage in their disease when CD4 counts in the majority were $<0.1 \times 10^{9} / 1$, it is not suprising that this was the commonest infection recorded. In Hickey's review of septicaemia in 131 AIDS patients, MAI was the organism most frequently isolated. ${ }^{5}$

Excluding infection due to MAI, patients with respiratory tract infection, followed by gastrointestinal tract infection, urinary tract 
infection and bacteraemia were most numerous. If episodes of infection per patient at risk per year are considered, the highest rates (due to recurrent infection) were recorded in the respiratory or urinary tracts. In a review of 444 AIDS patients by Rolston et al, ${ }^{2} 136$ episodes of bacterial infection were recorded (a rate of 31 episodes per 100 admissions) with bacteraemia the commonest infection. In Rolston's review Gram-negative aerobic bacilli caused $51 \%$ of bacterial infections and Gram-positives $46 \%$, four infections being polymicrobial. We also found an excess of Gram-negative infections over Gram-positive ones but less than $10 \%$ of infections in Rolston's review were community-acquired in contrast to our findings, where the majority were communityacquired. Rolston ${ }^{2}$ also found that overall the organisms most frequently isolated were $S$ aureus, $S$ epidermidis, Pseudomonas $\mathrm{sp}$ and $E$ coli. In our study, after MAI infection, more patients had $S$ aureus, $S$ pneumoniae, Pseudomonas $\mathrm{sp}$ and Campylobacter $\mathrm{sp}$ than other organisms, but if episodes of infection are considered, the highest rates were caused by $H$ influenzae, S pneumoniae and Pseudomonas sp.

In this review (excluding MAI) $S$ aureus and $P$ s aeruginosa were more frequently isolated as causes of bacteraemia than the other organisms (although numbers are small) but overall in bacteraemia, Gram-positive organisms predominated. The commonest source of bacteraemia appeared to be related to a Hickman line. Common causes of bacteraemia in AIDS patients include $S$ aureus, $S$ pneumoniae, $E$ coli, Salmonella sp, $H$ influenzae and $S$ epidermidis. ${ }^{1}$ In Hickey's review of AIDS patients with nonmycobacterial septicaemias, ${ }^{5} 61 \%$ were associated with an intravenous access device and of these $28 \%$ were due to Gram-negative organisms. A large proportion of cases of septicaemia caused by Gram-negative organisms in AIDS patients with no intravenous access device were due to Salmonella $\mathrm{sp}$ or Shigella $\mathrm{sp}$ and this was at a higher rate than in the normal population. They found a rate of 44 septicaemic episodes per $1000 \mathrm{HIV}$ or AIDS admissions, the majority of which were community-acquired.

More of the patients in this review had Campylobacter infection than Salmonella or Shigella. Bacterial diarrhoeal pathogens are more common in patients with HIV than in the rest of the population. ${ }^{1}$ Nontyphoidal salmonellas can present with enteric fever-like illness and disseminated salmonella is 20 times more frequent in HIV patients than in the normal population. ${ }^{1}$ Many HIV-positive patients can have severe complications and prolonged disease with recurrences and relapses despite appropriate therapy. ${ }^{6}$ Although Campylobacter can cause bacteraemia, become chronic or relapse, ${ }^{6}$ this did not occur in our patients.

Skin and soft tissue infections, as in other immunocompromised patients, may require long-term therapy, as did the patient with ecthyma gangrenosum. Three Hickman-related infections were due to Ps aeruginosa and on two occasions there was an associated bacteraemia. Central venous catheters are useful for nutrition, therapy and administration of blood products in these patients but infection with the risk of bacteraemia is a major hazard. The risk increases with previous catheter infection and advanced AIDS. ${ }^{7}$ Skoutelis $^{7}$ found Gram-positive cocci to predominate in these infections but where there were Gram-negatives, Pseudomonas sp was the most common.

Eleven of our patients had respiratory tract infections, of whom five had recurrent infection with $H$ influenzae, $S$ pneumoniae and $P_{S}$ aeruginosa, only two episodes of infection were nosocomial.

Between two and $10 \%$ of pneumonia in AIDS patients is caused by encapsulated bacteria. ${ }^{8}$ The predominant organisms include $S$ pneumoniae, $H$ influenzae, other streptococci and $M$ cattarhalis. ${ }^{8}$ Nosocomial bacterial pneumonia accounts for $10-15 \%$ of all bacterial pneumonia in AIDS-related complex or AIDS and is more likely to be Gram-negative. ${ }^{1}$ In AIDS patients with pneumococcal pneumonia $50-80 \%$ develop bacteraemia and with $H$ influenzae pneumonia, 5-25\% develop bacteraemia. ${ }^{8}$ Recurrences occur, presumably due to lack of production of antibody. The response to pneumococcal vaccine varies and is worth considering if the CD4 is greater than 450 but for those with advanced disease protective antibody levels may not develop. ${ }^{1}$ The use of prophylactic antibiotics ${ }^{1,8}$ for selected patients can be considered after one or two episodes of pneumococcal pneumonia but no trials of this have been performed. In HIV patients Hib vaccine has been tried but studies are needed to evaluate its use. ${ }^{6}$

Although probably an underestimate, only four of our patients were neutropenic but presumably many more had impaired neutrophil function. While neutropenia is an important factor leading to Gram-negative bacterial infection, Krumholtz ${ }^{9}$ found more Gram-positive infections in neutropenic AIDS patients who developed community-acquired bacteraemia.

We have reviewed the rates of bacterial infection in these severely immunocompromised patients and compared the rates between the different sites of infection and organisms causing infection. Although there are many other causes of infection the diversity and frequency of bacterial causes need to be considered in the patient with AIDS who presents with a febrile illness.

\section{Summary points}

- bacterial infections in AIDS patients are common

- the majority of these infections are community acquired

- bacterial infections in AIDS patients generally respond well to antimicrobial therapy but there is a high recurrence rate, particularly in the respiratory tract, which is the commonest site of infection

- after MAI, $H$ influenzae, $S$ pneumoniae and Pseudomonas sp were more frequently isolated than other organisms, often these same organisms caused recurrent chest infection 
1 Fraser V. Bacterial infections and AIDS. Missouri Med 1990; 87: $221-4$.

2 Rolston KVI, Radentz S, Rodriguez S. Bacterial and fungal infections in patients with the acquired immunodefiency syndrome. Cancer Detect Prevent 1990; 14: 377-81.

3 MaCarthy GA, Mercey D. The changing clinical features of HIV-1 infection in the United Kingdom. Commun Dis Rep $1994 ; 4$ (5): $53-8$

4 Horsburgh CR. Mycobacterium avium complex infection in the acquired immunodeficiency syndrome. $N$ Engl $7 \mathrm{Med}$ 1991; 324: $1332-8$

5 Hickey MM, Shanson DC. Septicaemia in patients with and without AIDS at Westminster Hospital, London. $\mathcal{F}$ Infect 1993; 27: $243-50$.
6 Fish DN, Danziger LH. Neglected pathogens: bacterial infections in persons with human immunodeficiency virus infections in persons with human immunodefici

7 Skoutelis AT, Murphy RL, MacDonell KB, VonRoenn JH, Sterkel CD, Phair JP. Indwelling central venous catheter infections in patients with acquired immune deficiency syndrome. F Acquir Immune Defic Syndr 1990; 3: 335-42. 8 Chaisson RE. Bacterial pneumonia in patients with human immunodeficiency virus infection. Semin Respir Infect 1989; 4: $133-8$.

9 Krumholz HM, Sande MA, Lo B. Community acquired bacteraemia in patients with acquired immunodeficiency bacteram in syndrome: clinical presentation, bacteriology and outcome. Am f Med 1989; 86: 776-9. 